\title{
Fast magnetoacoustic waves in curved coronal loops
}

\section{Trapped and leaky modes}

\author{
E. Verwichte, C. Foullon, and V. M. Nakariakov
}

Department of Physics, University of Warwick, Coventry CV4 7AL, UK

e-mail: Erwin. Verwichte@warwick. ac .uk

Received 1 August 2005 / Accepted 15 September 2005

\section{ABSTRACT}

\begin{abstract}
A study of vertically polarised fast magnetoacoustic waves in a curved coronal loop is presented. The loop is modeled as a semi-circular magnetic slab in the zero plasma- $\beta$ limit. The governing equations for linear waves are derived. We show that the wave mode behaviour depends on the slope of the equilibrium density profile, which is modeled as a piece-wise continuous power law curve of index $\alpha$. For all profiles, except for $\alpha=-4$, wave modes are not trapped in the loop and leak out into the external medium through wave tunneling. The particular case of $\alpha=-4$, which corresponds to a linearly increasing Alfvén speed profile, is examined in more detail as this is the only model that can support trapped wave modes. We compare the results with a straight slab model and find similar behaviour. Coupling between sausage and kink wave modes has not been found in the model.
\end{abstract}

Key words. Sun: oscillations - magnetohydrodynamics (MHD)

\section{Introduction}

The foundations of the coronal MHD wave theory have been established in the past decades (e.g. Edwin \& Roberts 1982, 1983; Heyvaerts \& Priest 1983; Hollweg \& Yang 1988; Sakurai et al. 1991), at a time when direct observational evidence of coronal wave activity was practically absent. Thus, theoretical models were designed to be simple but sufficient to describe the basic coronal wave dynamics: one-dimensionally structured straight waveguides with equilibrium quantities being either piece-wise constant or varying only in a thin layer. Nonetheless, the theory has been very successful in identifying the various MHD wave modes in current observations, by contemporary space missions such as SoHO and TRACE, and has given birth to the new technique of MHD coronal seismology (see e.g. Nakariakov \& Verwichte 2005).

In particular, fast magnetoacoustic waves have been observed with spatial resolution in the EUV in coronal loops as kink oscillations (Aschwanden et al. 1999; Nakariakov et al. 1999) and in radio as sausage oscillations (Nakariakov et al. 2003). Propagating fast waves have been observed using ground-based eclipse observations (Williams et al. 2001) and in open magnetic structures in the EUV (Verwichte et al. 2005). Of particular interest is the observation by Wang \& Solanki (2004) of a vertically polarised kink loop oscillation. This polarisation mode is resported to be strongly damped and to be associated with a density perturbation in anti-phase with the displacement. This feature cannot be explained with the straight loop model where the linear kink oscillation is practically incompressible.

The observations in turn offer the opportunity to construct more sophisticated coronal loop models, whose predictions can be directly tested against observational data. For instance, models have been extended to include effects such as fieldaligned structuring (e.g. Díaz et al. 2004; Andries et al. 2004; Mendoza-Briceño et al. 2004; Dymova \& Ruderman 2004) and loop curvature (e.g. Cargill et al. 1994; Smith et al. 1997; Miyagoshi et al. 2004; VanDoorselaere et al. 2004; Brady \& Arber 2005; Murawski et al. 2005; Selwa et al. 2005). The measure for the importance of loop curvature is the ratio of the loop cross-section radius and the radius of curvature. For typical coronal loops this ratio is typically less than 0.2 . Therefore, loop curvature seems to be of secondary importance for loop oscillations. In a curved geometry, though, the equilibrium quantities cannot be all kept constant (e.g. radially dependent magnetic field) and this affects the propagatory behaviour of the wave modes depending on the form of the Alfvén speed profile.

Cargill et al. (1994) studied oscillations in curved currentcarrying coronal loops. The combined effect of curvature and twist gives rise to an additional restoring force, known as the "hoop force". In response to the injection of magnetic flux into a loop, the presence of the hoop force was shown to give rise to a new oscillation mode, which manifests itself as the periodic change of the radius of curvature and the loop density. The oscillation frequencies obtained were significantly different from the frequencies of straight cylinder eigenmodes and 
were independent of the loop length. Roberts (2000) pointed out that it is likely that the effect of loop curvature would couple fast sausage and kink oscillations and hence blur the distinction between the two types of oscillations. This would mean that the fast kink oscillation could become leaky through the coupling with a leaky sausage wave. A detailed analytical calculation of this effect, though, is missing. VanDoorselaere et al. (2004) studied analytically the effect of loop curvature in a three-dimensional toroidal geometry for large thin loops. They focused in particular on the resonant damping of quasimodes and concluded that the effect of curvature is secondary. Also, they concluded that the different polarisation modes of the kink oscillation have the same behaviour. This, though, is in contradiction with the observations by Wang \& Solanki (2004).

Smith et al. (1997), Brady \& Arber (2005) and Selwa et al. (2005) have performed numerical simulations of fast magnetoacoustic waves in a density enhanced curved loop, embedded in a two-dimensional potential coronal arcade, with different height-dependent Alfvén speed profiles. Smith et al. (1997) studied the leakage of fast sausage and kink oscillations with an exponentially increasing Alfvén speed profile and came to the counterintuitive conclusion that the rate of leakage is inversely proportional to the wave period. Brady \& Arber (2005) pointed out that this result is probably a direct consequence of the proximity of the numerical boundaries to the modeled loop. Brady \& Arber (2005) studied the leakage of fast kink oscillations, initially excited by footpoint driving, with an inverse linear Alfvén speed profile. They found that the oscillation damping due to leakage is of the same order as observed kink oscillation damping times and that the rate of leakage is proportional to the wave period. Furthermore, they explained the leakage as wave tunneling through an evanescent barrier above the coronal loop. Unfortunately, because of the excitation mechanism, the leakage of the low oscillation harmonics could not be investigated. Selwa et al. (2005) studied impulsively excited fast magnetoacoustic oscillations with an exponentially decreasing Alfvén speed profile, in a bid to reproduce observational signatures. Besides an order of magnitude agreement with the observed damping times, they confirmed that the kink oscillation is compressive and that density perturbation is in anti-phase with the loop displacement. They also reported the presence of a leaky fast magnetoacoustic sausage mode, which they believe may play a role in explaining the observed damping.

Miyagoshi et al. (2004) have performed three-dimensional simulations of horizontally polarised fast magnetoacoustic waves in a potential arcade, triggered by a velocity field localised near the top of a magnetic flux tube. They concluded that the damping of the oscillation is explained as energy transport by fast magnetoacoustic waves propagating away from the excited fieldlines. They also concluded that an initially imposed density enhancement in the flux tube, which is not in equilibrium, does not substantially enhance the trapping of the excited waves. This result, though, is based only on three choices of initial density contrast.

The aim of this paper is to study analytically the fast magnetoacoustic wave modes in a two-dimensional curved slab model. Because fast magnetoacoustic kink waves modeled in a straight loop are nearly incompressible in the long wavelength limit, we wish to examine how the compressibility changes in curved loops and compare the results with observations. In particular, we shall examine the case of a linearly increasing Alfvén speed profile, which has interesting features that can be compared with wave modes in a straight loop model.

The paper is structured as follows. In Sect. 2, the curved loop model for a general Alfvén speed profile is presented. In Sect. 3, the equations governing the wave behaviour are derived. In Sect. 4, the wave mode solutions for the model with a piece-wise linear Alfvén speed profile are examined and compared with the solutions of a straight loop model in Sect. 5. In Sect. 6, the applicability of our model to observations is discussed. Finally, in Sect. 7, our main findings are summarised.

\section{Coronal loop model}

The effect of curvature on wave modes is studied through a semi-circular slab model, which corresponds to a description of a coronal arcade. Let $\mathbf{1}_{r}, \mathbf{1}_{\phi}$ and $\mathbf{1}_{z}$ represent the cylindrical coordinate unit vectors. The slab and the equilibrium magnetic field are directed along the azimuthal $\phi$-direction, with the solar surface located at $\phi=[0, \pi]$. The $z$-direction is parallel to the solar surface and perpendicular to the loop axis.

The curved magnetic slab, with a half-width $a$, and radius of curvature $R$, is line-tied at the solar surface with rigid boundary conditions (Rosner et al. 1984). Thus the semi-infinite space is partitioned into a lower external $(r<R-a)$, internal $(R-a \leq r \leq R+a)$ and upper external $(r>R+a)$ regions (see Fig. 1). The equilibrium quantities depend only on the radial coordinate. This assumption has two direct consequences. Firstly, the loop model essentially represents an arcade stretching in the $z$-direction. Secondly, as equilibrium quantities are not allowed to vary along the magnetic field, this model precludes the inclusion of gravity. A curved loop model with gravity is inherently two-dimensional. Although the effect of gravity is of interest, at this stage we wish to keep the model as simple as possible and focus on the effect of curvature on wave modes. For the same reason equilibrium flows are also not taken into account. Furthermore, since we are interested in fast magnetoacoustic waves, and the plasma- $\beta$ in the solar corona is much less than unity, it is reasonable to consider the zero plasma- $\beta$ limit.

For the condition of equilibrium, only the Lorentz force is present and needs to be zero, which for the considered model needs to be a potential magnetic field, $B_{0 \phi}(r) \mathbf{1}_{\phi}$, that obeys the relation:

$\frac{B_{0 \phi}}{r \mu_{\mathrm{o}}} \frac{\mathrm{d}}{\mathrm{d} r}\left(r B_{0 \phi}\right)=0 \quad$ or $\quad B_{0 \phi}=B_{0}\left(\frac{r}{R}\right)^{-1}$,

where $B_{0}$ is the field strength at $r=R$ and $\mu_{\mathrm{o}}$ is the permeability of a vacuum. Equilibrium quantities are denoted with a subscript "0".

The equilibrium density $\rho_{0}$ is an arbitrary function of $r$. It is enhanced in the internal region compared with the external regions to reflect the presence of a coronal loop:

$\rho_{0}(r)= \begin{cases}\rho_{0 \mathrm{i}}\left(\frac{r}{R}\right)^{\alpha} & |r-R| \leq a \\ \rho_{0 \mathrm{e}}\left(\frac{r}{R}\right)^{\alpha} & |r-R|>a,\end{cases}$ 


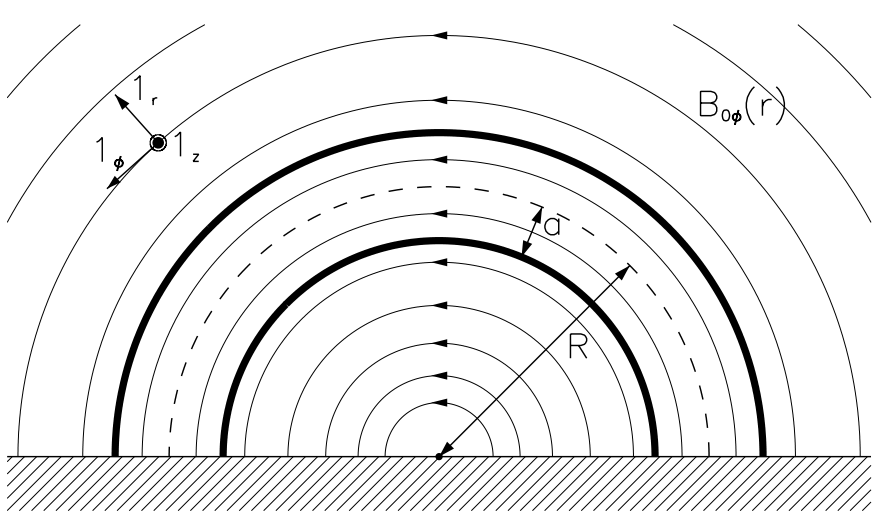

Fig. 1. Model of a curved coronal loop as a two-dimensional, semicircular, magnetic slab.

where the constants $\rho_{0 \mathrm{i}}$ and $\rho_{0 \mathrm{e}}$ are the values at $r=R$ of the internal and external density profiles respectively. The power law index $\alpha$ is the parameter that characterises the density profile. In principle, the value of $\alpha$ may differ in the internal and external regions and a similar analysis as presented here may be done. The ratio

$\chi=\frac{\rho_{0 \mathrm{e}}}{\rho_{0 \mathrm{i}}}$,

is a measure of the density contrast between the external and internal regions. For coronal loops $0<\chi<1$. Equation (2) implies, using Eq. (1), that the Alfvén speed is of the form:

$V_{\mathrm{A}}(r)=\left\{\begin{array}{l}V_{\mathrm{Ai}}\left(\frac{r}{R}\right)^{-\frac{\alpha+2}{2}} \\ V_{\mathrm{Ae}}\left(\frac{r}{R}\right)^{-\frac{\alpha+2}{2}}\end{array}\right.$

$$
\begin{aligned}
& |r-R| \leq a \\
& |r-R|>a,
\end{aligned}
$$

where the constants $V_{\mathrm{Ai}}$ and $V_{\mathrm{Ae}}$ are the values of the Alfvén speed at $r=R$ for the internal and external density profiles respectively.

\section{Governing equations}

In the cylindrical system $(r, \phi, z)$, we consider the ideal, gravitationless, zero plasma- $\beta$ MHD equations, which are linearised with respect to small perturbations from the equilibrium and written in function of the radial displacement, $\xi_{r}$, and the total pressure perturbation, $P$. We consider transverse perturbations, which are line-tied at the solar surface. These are essentially vertically polarised fast magnetoacoustic perturbations. In general, the magnetoacoustic and Alfvén wave modes are coupled in order to fulfil the line-tied condition at the solar surface (Goedbloed \& Halberstadt 1994), so that fast magnetoacoustic modes cannot be described separately. In our model the equilibrium magnetic field does not have a $z$-component (equivalent to magnetic shear in a loop arcade), which decouples Alfvén and magnetoacoustic waves. Additionally, by considering the zero plasma- $\beta$ limit, the slow magnetoacoustic waves are discarded. Therefore, a separate analysis of vertically polarised fast magnetoacoustic perturbations is possible in our model. We look for solutions of the form $\xi_{r}=\hat{\xi}_{r}(r) \sin (m \phi) \exp (-\mathrm{i} \omega t)$ and $P=\hat{P}(r) \sin (m \phi) \exp (-\mathrm{i} \omega t)$, where $\omega$ is the angular frequency of a mode of azimuthal degree $m$.
The set of equations describing fast magnetoacoustic waves of this type are derived in Appendix A, and are of the form

$$
\begin{aligned}
\frac{\mathrm{d}}{\mathrm{d} r}\left(\frac{\hat{\xi_{r}}}{r}\right) & =-\frac{1}{2 r} \frac{\hat{P}}{P_{0}}, \\
\frac{\mathrm{d}}{\mathrm{d} r}\left(\frac{\hat{P}}{P_{0}}\right) & =2 r\left(\frac{\omega^{2}}{V_{\mathrm{A}}^{2}(r)}-\frac{m^{2}}{r^{2}}\right) \frac{\hat{\xi}_{r}}{r},
\end{aligned}
$$

where $P_{0}=B_{0 \phi}^{2} / 2 \mu_{\mathrm{o}}$ is the equilibrium magnetic pressure. Eliminating $\hat{P}$ in Eqs. (5) and (6) yields the second order ordinary differential equation

$$
\left[\frac{\mathrm{d}^{2}}{\mathrm{~d} r^{2}}+\frac{1}{r} \frac{\mathrm{d}}{\mathrm{d} r}+\left(\frac{\omega^{2}}{V_{\mathrm{A}}^{2}(r)}-\frac{m^{2}}{r^{2}}\right)\right]\left(\frac{\hat{\xi}_{r}}{r}\right)=0,
$$

which is known as the Hain-Lust equation for this particular model. For constant Alfvén speed Eq. (7) reduces to a Bessel differential equation.

Two sets of boundary conditions are imposed, depending on the propagatory nature of the solution at $r=0$ and $r \rightarrow \infty$. If the solution is evanescent at $r=0$, we impose the condition that $\hat{\xi}_{r}$ vanishes at that point. In the limit of $r \rightarrow \infty$ an evanescent solution needs to have a vanishing kinetic energy density perturbation $\rho_{0}\left(\frac{\partial \xi_{r}}{\partial t}\right)^{2} / 2$. On the other hand, if the solution is oscillatory at $r=0(r \rightarrow \infty)$, we only permit a solution that propagates in the negative (positive) direction, away from the slab. Because the Alfvén and phase speeds are zero at $r=0$, a downward propagating wave would not be able to reflect but would accumulate in that point. At the interfaces between the external and internal regions, i.e. at $r=R \pm a$, the radial displacement and total pressure perturbation need to be continuous.

The longitudinal oscillation phase speed, $V_{\mathrm{ph}}$, in a curved slab model is the ratio of the frequency, $\omega$, and the wave vector component in the direction of the azimuthal magnetic field, $m / r$, i.e. $V_{\mathrm{ph}}(r)=\omega r / m$. This means that the phase speed depends linearly on $r$. The sign of the third term in Eq. (7) dictates the propagatory nature of the solution. Where $V_{\mathrm{ph}}(r)>V_{\mathrm{A}}(r)$, this term is positive and the solution is oscillatory. On the other hand, where $V_{\mathrm{ph}}(r)<V_{\mathrm{A}}(r)$, this term is negative and the solution is evanescent. This is equivalent to considering the slope of the Alfvén frequency profile, $\omega_{\mathrm{A}}=V_{\mathrm{A}} m / r$. For an Alfvén speed profile of the form (4), there are three possible scenarios:

a. $\alpha<-4, \mathrm{~d} \omega_{\mathrm{A}} / \mathrm{d} r>0$ : the solution is always oscillatory for small $r$ and evanescent for large $r$.

b. $\alpha=-4, \mathrm{~d} \omega_{\mathrm{A}} / \mathrm{d} r=0$ : the solution is either oscillatory or evanescent, depending whether the phase speed is above or below the Alfvén speed profile.

c. $\alpha>-4, \mathrm{~d} \omega_{\mathrm{A}} / \mathrm{d} r<0$ : the solution is always evanescent for small $r$ and oscillatory for large $r$.

Consider a solution that is oscillatory in at least part of the internal region, then for $\alpha>-4$ the solution is evanescent in the upper region up to a certain distance where the solution becomes oscillatory again. This scenario is consistent with the concept of wave tunneling. Similarly, wave tunneling occurs if $\alpha<-4$, but into the lower external region. Brady \& Arber (2005) studied the case $\alpha=0$ numerically and found that fast 
magnetoacoustic kink oscillations leak into the upper external region by wave tunneling through the evanescent barrier.

Another manner to distinguish between the curved slab models is the density perturbation, which is found from the mass continuity Eq. (A.1), using Eqs. (2) and (A.8)

$$
\left(\frac{\rho}{\rho_{0}}\right)=\frac{1}{2} \frac{P}{P_{0}}-2 \frac{\xi_{r}}{r}-\frac{1}{\rho_{0}} \frac{\mathrm{d} \rho_{0}}{\mathrm{~d} r} \xi_{r}=\frac{1}{2} \frac{P}{P_{0}}-2 \frac{\xi_{r}}{r}-\alpha \frac{\xi_{r}}{r} .
$$

The density perturbation consists of three contributions. The first contribution is due to the magnetic pressure perturbation and this term also appears in the straight coronal loop model (Edwin \& Roberts 1982). The second contribution is due to the loop curvature and is associated with the loop length perturbation. We can see that from calculating the linearised loop length perturbation of the loop axis:

$$
\left(\frac{L}{L_{0}}\right)=\frac{1}{\pi R} \int_{0}^{\pi} \xi_{r}(R) \mathrm{d} \phi=\frac{2}{m \pi}\left(1-(-1)^{m}\right) \frac{\hat{\xi}_{r}(R)}{R} .
$$

Note that only odd harmonics perturb the loop length. The third contribution is due to the advection of the equilibrium density. This term is the difference between the Eulerian and the Langrangian description of the density perturbation. The latter two contributions compete with each other and the balance is determined by the sign of the factor $2+\alpha$, which, from examining Eq. (4), is equivalent to the sign of the slope of the Alfvén speed profile. Because both terms are proportional to the radial displacement, any uniform displacement of the whole loop in one direction (such as occurs approximately in kink mode oscillations) would result in an Eulerian density perturbation in phase or in anti-phase with this displacement, depending on the sign of the factor $2+\alpha$. There are three possible scenarios:

a. $\alpha<-2, \mathrm{~d} V_{\mathrm{A}} / \mathrm{d} r>0$ : the effect of density advection is stronger than the effect of loop curvature.

b. $\alpha=-2, \mathrm{~d} V_{\mathrm{A}} / \mathrm{d} r=0$ : the effects of loop curvature and density advection balance each other.

c. $\alpha>-2, \mathrm{~d} V_{\mathrm{A}} / \mathrm{d} r<0$ : the effect of density advection is weaker than the effect of loop curvature.

The Langrangian density perturbation is

$\left(\frac{\delta \rho}{\rho_{0}}\right)=\frac{\rho}{\rho_{0}}+\frac{1}{\rho_{0}}(\boldsymbol{\xi} . \boldsymbol{\nabla}) \rho_{0}=\frac{1}{2} \frac{P}{P_{0}}-2 \frac{\xi_{r}}{r}$,

which does not explicitly depend on the parameter $\alpha$. The Lagrangian density perturbation is always in anti-phase with the displacement.

In the remaining of this paper, we focus on fast magnetoacoustic wave modes for the model with a linear Alfvén speed profile, i.e. $\alpha=-4$. This is of particular interest because it is the only case where trapped wave modes are possible. We shall compare the results to a straight slab model with piecewise constant equilibrium quantities (Edwin \& Roberts 1982). The other Alfvén speed profiles are also of interest but we shall investigate them in a future paper.

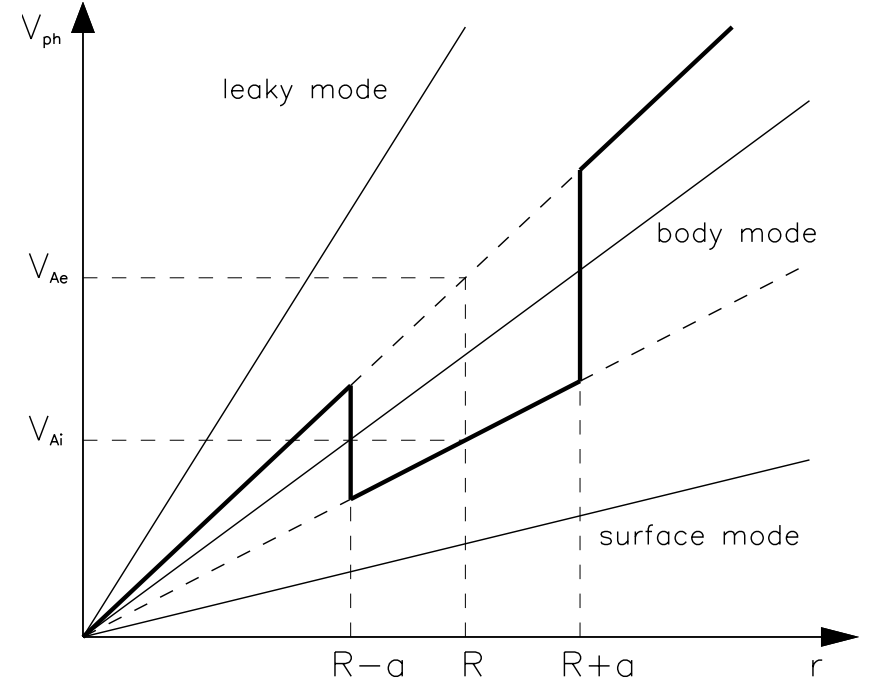

Fig. 2. The piece-wise linear Alfvén speed profile as a function of $r$, corresponding to Eq. (4) with $\alpha=-4$. The phase speeds of three wave scenarios corresponding to surface, body and leaky modes are shown.

\section{Wave modes in a model with a piece-wise linear Alfvén speed profile}

With a piece-wise linear Alfvén speed profile, corresponding to $\rho_{0} \sim r^{-4}$, Eq. (7) reduces to

$\left[\frac{\mathrm{d}^{2}}{\mathrm{~d} r^{2}}+\frac{1}{r} \frac{\mathrm{d}}{\mathrm{d} r}-\frac{\kappa_{\{\mathrm{i}, \mathrm{e}\}}^{2}}{r^{2}}\right]\left(\frac{\hat{\xi}_{r}}{r}\right)=0$,

where

$\kappa_{\mathrm{i}}^{2}=m^{2}-\Omega^{2}$ and $\kappa_{\mathrm{e}}^{2}=m^{2}-\chi \Omega^{2}$,

and $\Omega=\omega R / V_{\mathrm{Ai}}$ is a dimensionless frequency. Equation (11) has solutions of the form

$\left(\frac{\hat{\xi_{r}}}{r}\right)=\sum_{m=1}^{\infty} A_{m}\left(\frac{r}{R}\right)^{\kappa_{\mathrm{i}, \mathrm{e}\}}}+B_{m}\left(\frac{r}{R}\right)^{-\kappa_{\{\mathrm{i}, \mathrm{e}\}}}$,

where $A_{m}$ and $B_{m}$ are integration constants. The sign of $\kappa_{\{\mathrm{i}, \mathrm{e}\}}^{2}$ determines whether the solution is oscillatory (negative) or evanescent (positive). The condition $V_{\mathrm{Ae}}>V_{\mathrm{Ai}}$, or $\kappa_{\mathrm{i}}^{2}<\kappa_{\mathrm{e}}^{2}$, allows three possible scenarios:

a. Surface modes: $0<\kappa_{\mathrm{i}}^{2}<\kappa_{\mathrm{e}}^{2}$, the solution is evanescent everywhere.

b. Body modes: $\kappa_{\mathrm{i}}^{2}<0<\kappa_{\mathrm{e}}^{2}$, the solution is oscillatory inside the loop, but is evanescent in the external regions.

c. Leaky modes: $\kappa_{\mathrm{i}}^{2}<\kappa_{\mathrm{e}}^{2}<0$, the solution is oscillatory everywhere.

The surface and body (trapped) modes are evanescent in the external regions. Therefore, the boundary conditions of vanishing $\hat{\xi}_{r}$ at $r=0$ and kinetic energy density perturbation at $r \rightarrow \infty$ need to applied. For the leaky modes, the condition of outward propagating solutions need to be applied. In that case we expect $\omega$ to be complex with a negative imaginary part, to describe the draining of wave energy from the loop. In this way, the curved slab with a piece-wise linear Alfvén speed profile supports the same types of modes as in the straight slab model. 


\subsection{Trapped modes}

Trapped modes $\left(\kappa_{\mathrm{e}}^{2}>0\right)$ for a given degree $m$, which obey the boundary conditions at $r=0$ and $r \rightarrow \infty$, have the radial displacement

$\left(\frac{\hat{\xi}_{r}}{r}\right)= \begin{cases}A_{m \mathrm{e}}\left(\frac{r}{R}\right)^{\left|\kappa_{\mathrm{e}}\right|} & r<R-a \\ A_{m \mathrm{i}}\left(\frac{r}{R}\right)^{K_{\mathrm{i}}}+B_{m \mathrm{i}}\left(\frac{r}{R}\right)^{-\kappa_{\mathrm{i}}} & |r-R| \leq a \\ B_{m \mathrm{e}}\left(\frac{r}{R}\right)^{-\left|\kappa_{\mathrm{e}}\right|} & r>R+a,\end{cases}$

and magnetic pressure perturbation (using Eq. (5))

$\left(\frac{\hat{P}}{P_{0}}\right)= \begin{cases}-2\left|\kappa_{\mathrm{e}}\right| A_{m \mathrm{e}}\left(\frac{r}{R}\right)^{\left|\kappa_{\mathrm{e}}\right|} & r<R-a \\ -2 \kappa_{\mathrm{i}}\left[A_{m \mathrm{i}}\left(\frac{r}{R}\right)^{\kappa_{\mathrm{i}}}-B_{m \mathrm{i}}\left(\frac{r}{R}\right)^{-\kappa_{\mathrm{i}}}\right] & |r-R| \leq a \\ 2\left|\kappa_{\mathrm{e}}\right| B_{m \mathrm{e}}\left(\frac{r}{R}\right)^{-\left|\kappa_{\mathrm{e}}\right|} & r>R+a,\end{cases}$

where the real part of $\kappa_{\mathrm{i}}$ is positive, i.e. $\mathfrak{R} \kappa_{\mathrm{i}}>0$. The case $\left|\kappa_{\mathrm{e}}\right| \leq 1$ needs to be considered carefully. In the upper external region, both solutions for $\hat{\xi}_{r}$ (see Eq. (13)) actually have a kinetic energy density perturbation that decays to zero, eventhough $\hat{\xi}_{r}$ itself becomes infinite. Of the two solutions the one which grows faster than $V_{\mathrm{A}}$ is eliminated. In the lower external region, both solutions for $\hat{\xi}_{r}$ actually become zero at $r=0$, eventhough the total pressure perturbation, $\hat{P}$, becomes infinite. Of the two solutions the one which decays faster than $P_{0}$ is discarded. With these conditions, the solutions for the cases $\left|\kappa_{\mathrm{e}}\right| \leq 1$ and $\left|\kappa_{\mathrm{e}}\right|>1$ are of the same form and given by Eqs. (14) and (15).

Equating the solutions for $\hat{\xi}_{r}$ and the Lagrangian total pressure perturbation, $\delta \hat{P}=\hat{P}-2 P_{0} \hat{\xi}_{r} / r$, across the interfaces at $r=R \pm a$ leads to a dispersion relation

$\left(\frac{\left|\kappa_{\mathrm{e}}\right|-\kappa_{\mathrm{i}}}{\left|\kappa_{\mathrm{e}}\right|+\kappa_{\mathrm{i}}}\right)-\mathrm{e}^{\kappa_{\mathrm{i}} \ln q} \mathrm{e}^{-\mathrm{i} n \pi}=0$,

where

$q=\frac{R+a}{R-a}$,

and where $n$ is an integer. Each value of $n$ represents a different solution branch. Even (odd) values of $n$ refer to symmetric (asymmetric) solutions, i.e. $B_{m \mathrm{i}}=-(-1)^{n} A_{m \mathrm{i}}$. The dispersion relation (16) can be rewritten as

$\left\{\begin{array}{l}\tanh \\ \operatorname{coth}\end{array}\right\}\left(\frac{1}{2} \kappa_{\mathrm{i}} \ln q\right)=-\frac{\kappa_{\mathrm{i}}}{\left|\kappa_{\mathrm{e}}\right|}$,

where solutions arising from the tanh and coth functions correspond to $n$ even and odd respectively. By analogy to the symmetric and asymmetric solutions in the straight slab wave problem, we call these solutions sausage and kink modes respectively (Edwin \& Roberts 1982). In contrast to the notation used in Edwin \& Roberts (1982), $m$ and $n$ here refer to the longitudinal (azimuthal) wavenumber and the mode branch number respectively.

\subsection{Leaky modes}

Leaky modes $\left(\kappa_{\mathrm{e}}^{2}<0\right)$ for a particular value of $m$, which obeys the boundary conditions at $r=0$ and $r \rightarrow \infty$ of outward progating solutions have radial displacement

$\left(\frac{\hat{\xi}_{r}}{r}\right)= \begin{cases}A_{m \mathrm{e}}\left(\frac{r}{R}\right)^{-\operatorname{sgn}\left(\mathfrak{I}_{\mathrm{e}}\right) \kappa_{\mathrm{e}}} & r<R-a \\ A_{m \mathrm{i}}\left(\frac{r}{R}\right)^{\mathrm{i}_{\mathrm{i}}}+B_{m \mathrm{i}}\left(\frac{r}{R}\right)^{-\mathrm{i} \kappa_{\mathrm{i}}} & |r-R| \leq a \\ B_{m \mathrm{e}}\left(\frac{r}{R}\right)^{\operatorname{sgn}\left(\mathfrak{J}_{\mathrm{e}}\right) \kappa_{\mathrm{e}}} & r>R+a,\end{cases}$

and magnetic pressure perturbation (using Eq. (5))

$$
\left(\frac{\hat{P}}{P_{0}}\right)= \begin{cases}2 \operatorname{sgn}\left(\mathfrak{J} \kappa_{\mathrm{e}}\right) \kappa_{\mathrm{e}} A_{m \mathrm{e}}\left(\frac{r}{R}\right)^{-\operatorname{sgn}\left(\mathfrak{J} \kappa_{\mathrm{e}}\right) \kappa_{\mathrm{e}}} & r<R-a \\ -2 \mathrm{i} \kappa_{\mathrm{i}}\left[A_{m \mathrm{i}}\left(\frac{r}{R}\right)^{\mathrm{i} \kappa_{\mathrm{i}}}-B_{m \mathrm{i}}\left(\frac{r}{R}\right)^{-\mathrm{i} \kappa_{\mathrm{i}}}\right] & |r-R| \leq a \\ -2 \operatorname{sgn}\left(\mathfrak{J} \kappa_{\mathrm{e}}\right) \kappa_{\mathrm{e}} B_{m \mathrm{e}}\left(\frac{r}{R}\right)^{\operatorname{sgn}\left(\mathfrak{J} \kappa_{\mathrm{e}}\right) \kappa_{\mathrm{e}}} & r>R+a,\end{cases}
$$

where $\mathfrak{J} \kappa_{\mathrm{e}}$ is the imaginary part of $\kappa_{\mathrm{e}}$. Equating the solutions for $\hat{\xi}_{r}$ and the Lagrangian total pressure perturbation, $\delta \hat{P}=\hat{P}-$ $2 P_{0} \hat{\xi}_{r} / r$, across the interfaces at $r=R \pm a$ leads to the dispersion relation

$\left(\frac{\operatorname{sgn}\left(\mathfrak{J} \kappa_{\mathrm{e}}\right) \kappa_{\mathrm{e}}-\mathrm{i} \kappa_{\mathrm{i}}}{\operatorname{sgn}\left(\mathfrak{J} \kappa_{\mathrm{e}}\right) \kappa_{\mathrm{e}}+\mathrm{i} \kappa_{\mathrm{i}}}\right)-\mathrm{e}^{-\mathrm{i} \kappa_{\mathrm{i}} \ln q} \mathrm{e}^{-\mathrm{i} n \pi}=0$,

where the even (odd) $n$ again refers to symmetric (asymmetric) solutions. Equation (21) can be rewritten as

$\left\{\begin{array}{l}\tanh \\ \operatorname{coth}\end{array}\right\}\left(\frac{1}{2} \mathrm{i} \kappa_{\mathrm{i}} \ln q\right)=\frac{\kappa_{\mathrm{i}}}{\operatorname{sgn}\left(\mathfrak{J} \kappa_{\mathrm{e}}\right) \kappa_{\mathrm{e}}}$,

where solution arising from the tanh and coth functions correspond to leaky sausage and kink modes respectively. The leaky solutions have perturbation amplitudes that grow with distance from the slab and become infinite at $r=0$ and $r \rightarrow \infty$. This is equivalent to an infinite wave amplitude inside the slab at time $t \rightarrow-\infty$.

\subsection{Eigenmode characteristics}

In addition to the azimuthal wave number $m$, the dispersion relations depend explicitly on only two other parameters: $\chi$, the density contrast and $a / R$, the ratio of the loop half-width and radius. For coronal loops both parameters lie between 0 and 1. For illustration, the left-hand sides of the dispersion relations (16) and (21) are evaluated in Fig. 3 for the case of kink modes (odd $n$ ), with $m=5, \chi=0.2$ and $a / R=0.2$. The circles highlight the location of roots. Besides one real root, there are two roots with negative imaginary parts, associated with leaky modes. It is interesting to note that roots with positive imaginary parts, indicative of unstable modes, are not present in the range covered.

Dispersion relations (16) and (21) are solved for $\Omega$ as a function of $m$. Figures 4 and 5 are dispersion diagrams showing respectively the real and imaginary parts of the solutions for the case $\chi=0.2$ and $a / R=0.2$. The dispersion diagrams show the same characteristic behaviour as in the straight slab model (Edwin \& Roberts 1982). There is an alternation of kink and sausage mode branches. In the short wavelength limit, when 

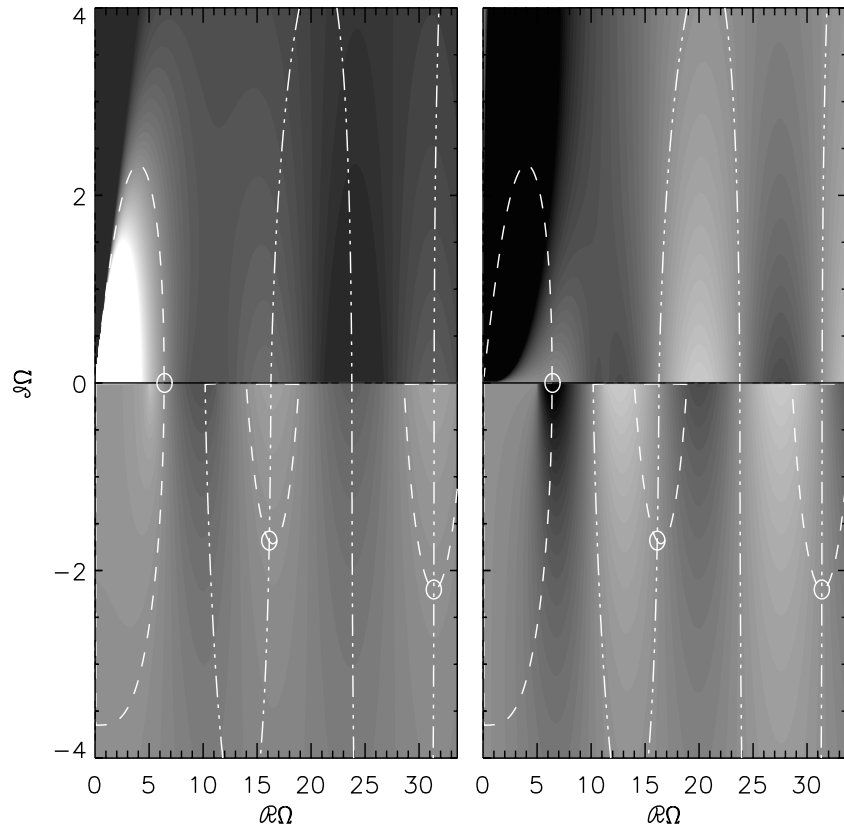

Fig. 3. Real (left) and imaginary (right) parts of an evaluation of the left-hand sides of dispersion relations (16) and (21) as a function of the real and imaginary part of $\Omega$, for kink modes $(m=5, \chi=0.2$ and $a / R=0.2$ ). Zeros in the real and imaginary parts are indicated by dashed and dot-dashed lines respectively. Intersections of the two types of curves, highlighted with a circle, indicate solutions to the dispersion relations.

$m a / R \gg 1$, all modes are trapped body modes, with phase speeds tending to the internal Alfvén speed. In the long wavelength limit, where $m a / R \ll 1$, the modes are leaky and the rate of leakage for all modes tend to the same maximum value (see Fig. 5). Therefore there exist a finite value of $m a / R$ for which there is a cut-off. The fundamental kink mode branch $(n=-1)$ is the one exception. This mode is always trapped and tends to the external Alfvén speed as $m a / R \rightarrow 0$. As in the straight coronal slab model, parallel propagating surface modes do not exist.

We shall examine three characteristics of the wave mode frequencies in more detail: the cut-offs, the long and the short wavelength limits. The branches of the trapped modes have cutoffs and become leaky for a certain minimum value of $m a / R$. Only the first branch of the kink mode, which has a cut-off at $m=0$ exists for all value of $m a / R$. At the cut-off, $\kappa_{\mathrm{e}}^{2}=0$, or $\Omega / m=\chi^{-1 / 2}$. Dispersion relation (21) then becomes:

$\mathrm{e}^{\mathrm{i} \mid k_{\mathrm{i}} \ln q} \mathrm{e}^{-\mathrm{i} n \pi}=-1$,

or

$m_{\mathrm{c}}=\frac{(2 n+1) \pi}{\ln q} \sqrt{\frac{\chi}{1-\chi}}$.

The location of the cut-off for the fundamental kink mode is found for $n=-1$ and is $m=0$. Figure 6 shows how Eq. (24) divides the parameter space of $a / R$ and $\chi$ into regimes where the first four harmonics of the fundamental sausage mode branch $(n=0)$ are either trapped or leaky. For comparison the curves for the straight slab model have been overplotted. It shows

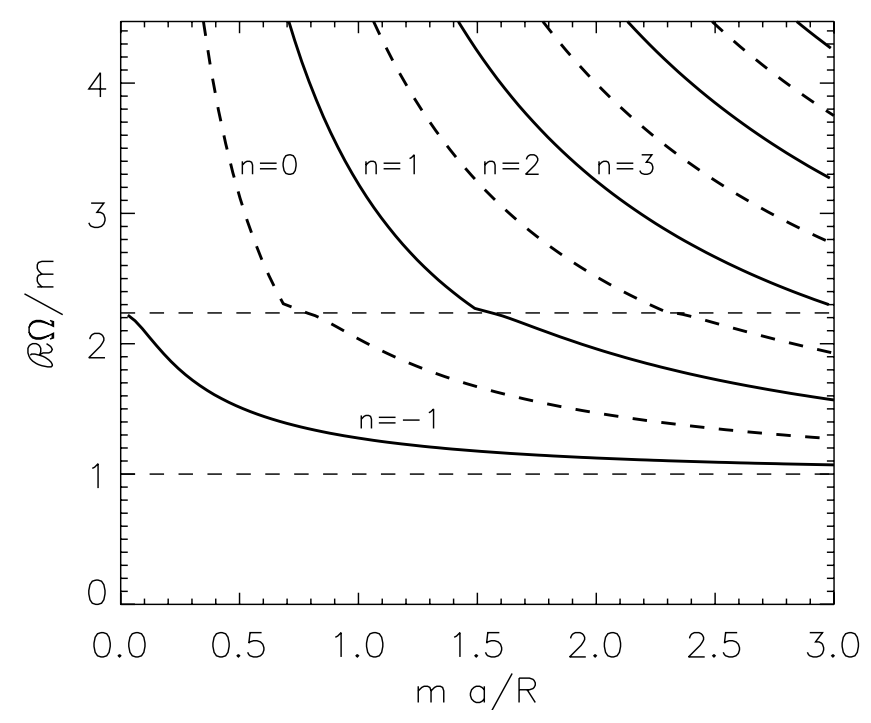

Fig. 4. Dispersion diagram showing the normalised phase speeds of the solutions of dispersion relations (16) and (21) as a function of $m a / R$. The thick solid and dashed lines are the solution branches for the kink and sausage modes respectively. The first 4 branches are annotated. The parameters $\chi=0.2$ and $a / R=0.2$. The position where $V_{\mathrm{ph}}(R)=V_{\mathrm{Ai}}$ and $V_{\mathrm{ph}}(R)=V_{\mathrm{Ae}}$ are shown with thin dashed lines. Where $V_{\mathrm{ph}}(R)$ is below (above) the value of $V_{\mathrm{Ae}}$, corresponding to $\kappa_{\mathrm{e}}^{2}>0\left(\kappa_{\mathrm{e}}^{2}<0\right)$, the wave modes are trapped (leaky).

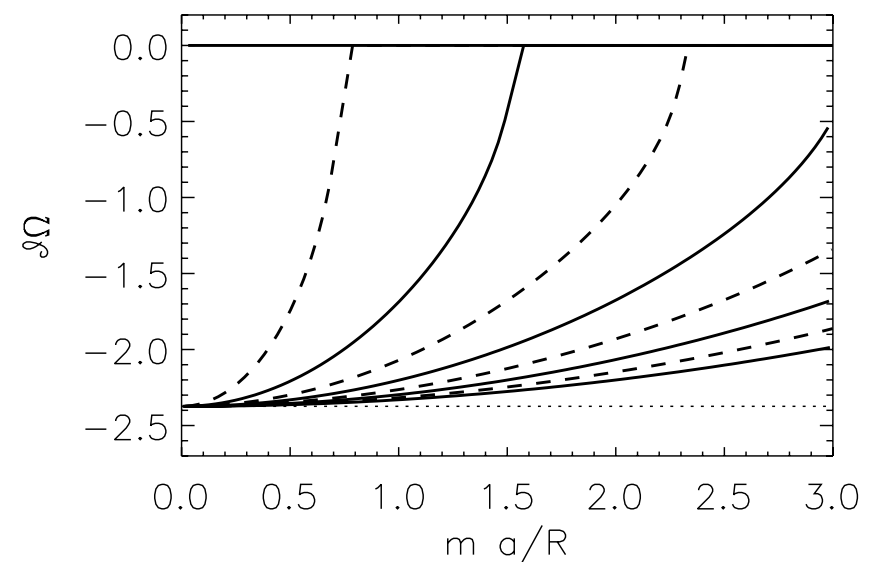

Fig. 5. Dispersion diagram showing the imaginary part of the wave mode frequency, $\mathfrak{J}(\Omega)$, of the solutions of dispersion relations (16) and (21) as a function of $m a / R$. The thick solid and dashed lines are the solution branches for the kink and sausage modes respectively. The first 4 branches are annotated. The parameters $\chi=0.2$ and $a / R=0.2$. The dotted line is the decay rate in the long wavelength limit given by Eq. (26).

that sausage modes are more easily trapped in the curved slab model presented here than in the straight slab model, with the differences becoming more pronounced for large values of $a / R$.

In the long wavelength limit $(\mathrm{ma} / R \ll 1)$ all modes except the fundamental kink mode are leaky. In this limit the dispersion relation (21) becomes approximately

$\mathrm{e}^{\mathrm{i} \Omega \ln q} \mathrm{e}^{-\mathrm{i} n \pi} \approx-\left(\frac{1+\sqrt{\chi}}{1-\sqrt{\chi}}\right)$ 


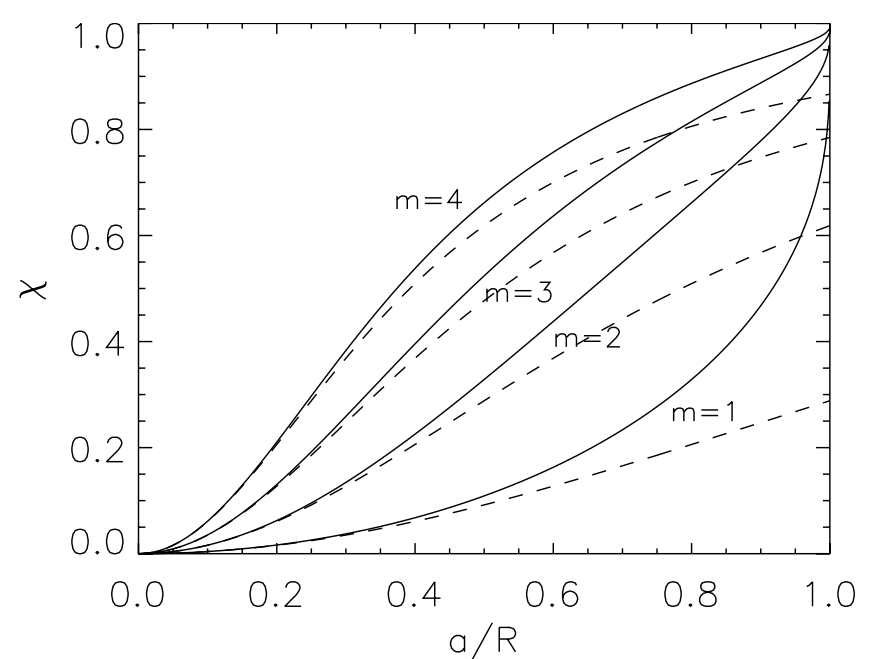

Fig. 6. Regimes in parameter space of $a / R$ and $\chi$ for which the sausage mode $(n=0)$ is trapped or leaky, for the fundamental mode $(m=1)$ and three harmonics. Below (above) the solid curve, described by Eq. (24), the mode is trapped (leaky). The dashed curves show the equivalent for the case of the straight slab.

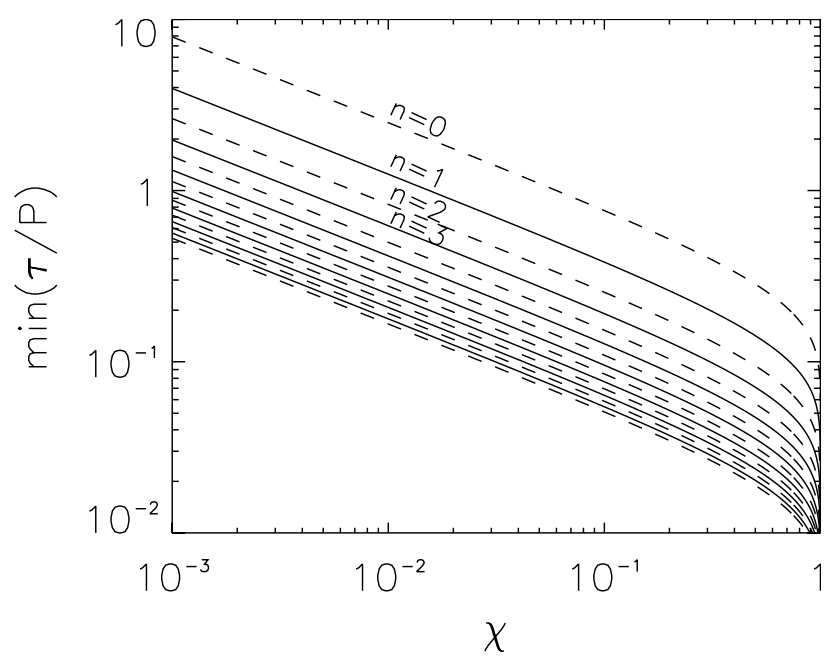

Fig. 7. Ratio of the e-folding time, $\tau$, and the period, $P$, for the first 15 leaky modes as a function of $\chi$ in the long wavelength limit ( $m a / R \ll 1)$ using Eq. (26).

which has solutions of the form

$\omega \approx \frac{V_{\mathrm{Ai}}}{R}\left[(n+1) \pi-\mathrm{i} \ln \left(\frac{1+\sqrt{\chi}}{1-\sqrt{\chi}}\right)\right] / \ln q$.

This formula is not valid for the fundamental kink mode $(n=-1)$, which is not leaky and is not described by Eq. (21). The imaginary part of Eq. (26) gives the maximum decay rate of the leaky mode and is the same for all leaky modes. Figure 7 shows the ratio of the maximum decay rate to the mode period, $\tau / P=\mathfrak{R} \Omega / 2 \pi \mathfrak{J} \Omega$. It gives an indication of the rate of leakage for low harmonic oscillations in large, thin coronal loops. For such a case, a leaky sausage mode $(n=0)$ may damp in only a few oscillation periods, unless the loop is overdense. Solutions associated with the higher solution branches damp even quicker.
In the long wavelength limit, the fundamental kink mode branch is not leaky and tends to the solution $\Omega=m / \sqrt{\chi}$. By substituting a solution of the form $\Omega=m\left(1-\delta \Omega m^{2}\right) / \sqrt{\chi}$, with $|\delta \Omega| \ll 1$, into Eq. (16) for trapped kink modes, we find the solution for $m a / R \ll 1$

$\omega \approx \frac{V_{\mathrm{Ai}} m}{R \sqrt{\chi}}\left[1-\frac{1}{8}\left(\frac{1-\chi}{\chi}\right)^{2} \ln ^{2} q m^{2}\right]$.

In the short wavelength limit $(m a / R \gg 1)$ all modes are trapped body modes with a phase speed $V_{\mathrm{ph}}(R)$ tending to $V_{\mathrm{Ai}}(\Omega \rightarrow m)$. Consider a solution of the form $\Omega=m+\delta \Omega$ with $|\delta \Omega| \ll 1$. In the short wavelength limit dispersion relation (16) becomes approximately

$\mathrm{e}^{\mathrm{i} \sqrt{2} \delta \Omega^{1 / 2} \ln q} \mathrm{e}^{-\mathrm{i} n \pi} \approx 1$.

Therefore in the short wavelength limit dispersion relation (16) has solutions which are approximately

$\omega \approx \frac{m V_{\mathrm{Ai}}}{R}+\frac{(n+2)^{2} \pi^{2} V_{\mathrm{Ai}}}{2 R \ln ^{2} q} \frac{1}{m}$.

\subsection{Eigenfunctions}

Figure 8 shows the wave solutions $\hat{\xi}_{r} / r, \hat{P} / P_{0}$ and the density perturbation $\hat{\rho} / \rho_{0}$ as a function of the radial coordinate for a kink body, sausage body and a sausage leaky mode. The kink mode is an asymmetric solution with real $A_{m \mathrm{i}}=B_{m \mathrm{i}}$. It has a nonzero displacement at the loop axis, situated at $r=R$ and displaces the whole loop in the transverse (radial) direction (see Fig. 9). The sausage mode is a symmetric solution with purely imaginary $A_{m \mathrm{i}}=-B_{m \mathrm{i}}$. The displacement at $r=R$ is zero so that the loop axis remains stationary (see Fig. 9). The example of the leaky mode shows the propagatory behaviour in the external regions. At $r=0$ the Alfvén and the phase speed become zero and the energy of the leaky wave propagating downwards accumulates there.

Of particular interest is the density perturbation. Consider Eq. (8) with $\alpha=-4$, then the solution for trapped modes is

$\left(\frac{\hat{\rho}}{\rho_{0}}\right)=\left\{\begin{array}{cc}\left(2-\left|\kappa_{\mathrm{e}}\right|\right) A_{m \mathrm{e}}\left(\frac{r}{R}\right)^{\left|\kappa_{\mathrm{e}}\right|} & r<R-a \\ \left(2-\kappa_{\mathrm{i}}\right) A_{m \mathrm{i}}\left(\frac{r}{R}\right)^{K_{\mathrm{i}}} & \\ +\left(2+\kappa_{\mathrm{i}}\right) B_{m \mathrm{i}}\left(\frac{r}{R}\right)^{-\kappa_{\mathrm{i}}} & |r-R| \leq a \\ \left(2+\left|\kappa_{\mathrm{e}}\right|\right) B_{m \mathrm{e}}\left(\frac{r}{R}\right)^{-\left|\kappa_{\mathrm{e}}\right|} & r>R+a .\end{array}\right.$

The density perturbation at $r=R$ for body modes is:

$\left(\frac{\hat{\rho}}{\rho_{0}}\right)(R)=2\left(A_{m \mathrm{i}}+B_{m \mathrm{i}}\right)-\mathrm{i}\left|\kappa_{\mathrm{i}}\right|\left(A_{m \mathrm{i}}-B_{m \mathrm{i}}\right)$,

where the two terms are the contributions from the displacement and magnetic pressure perturbations respectively. The equivalent Lagrangian density perturbation is

$\left(\frac{\delta \hat{\rho}}{\rho_{0}}\right)(R)=-2\left(A_{m \mathrm{i}}+B_{m \mathrm{i}}\right)-\mathrm{i}\left|\kappa_{\mathrm{i}}\right|\left(A_{m \mathrm{i}}-B_{m \mathrm{i}}\right)$.

For a kink mode $\hat{\rho} / \rho_{0}(R)=4 A_{m i}$, which is due to the displacement. This amplitude is four times that of $\hat{\xi}_{r} / r$ and does not 

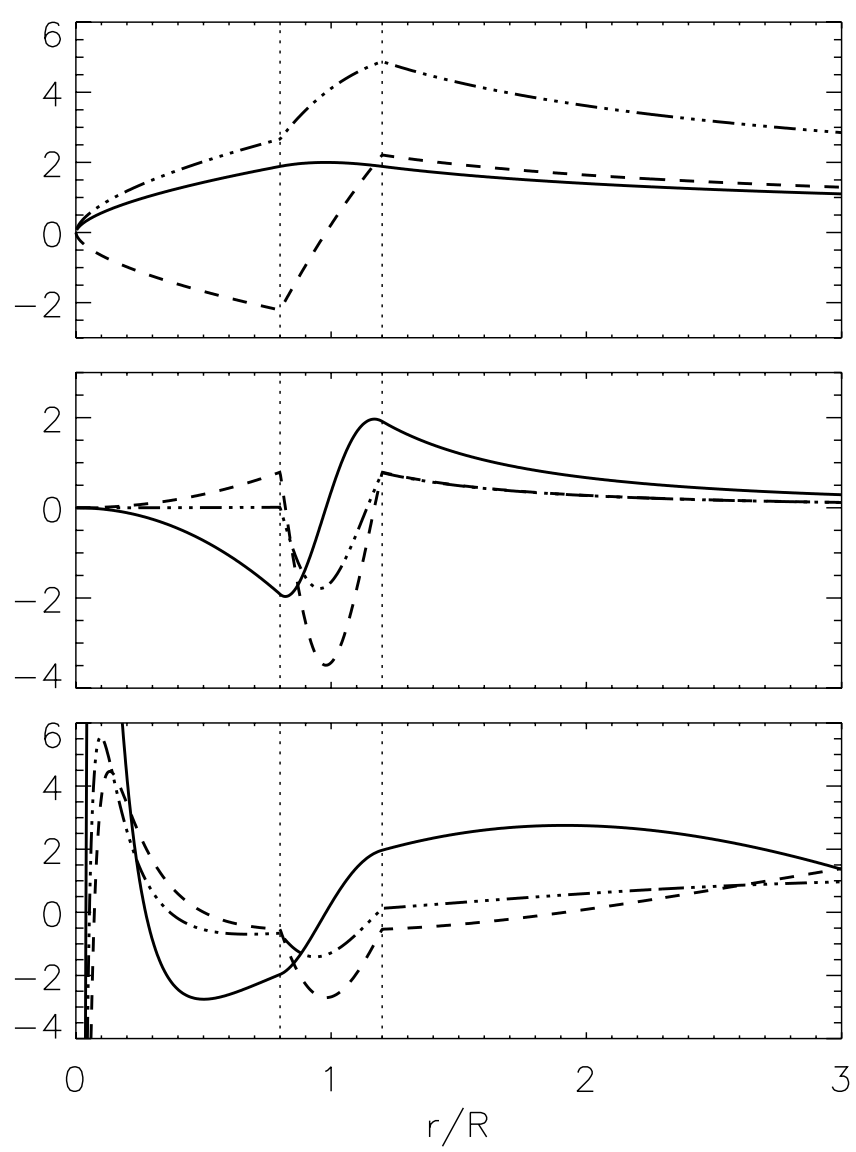

Fig. 8. The quantities $\hat{\xi}_{r} / r$ (solid line), $\hat{P} / P_{0}$ (dashed line) and $\hat{\rho} / \rho_{0}$ (dot-dashed line) as a function of radial distance $r / R$ for three wave modes: top: fundamental kink mode at $m a / R=0.21$, middle: trapped fundamental sausage mode at $m a / R=1.00$ and bottom: real part of the leaky fundamental sausage mode at $m a / R=0.65$. The amplitudes of the wave modes are $A_{\mathrm{i}}=B_{\mathrm{i}}=1$ and $A_{\mathrm{i}}=-B_{\mathrm{i}}=\mathrm{i}$ for the kink and sausage modes respectively. In the two latter plots the magnetic pressure and density perturbations have been divided by a factor 10 . The parameters $\chi=0.2$ and $a / R=0.2$.

depend on the phase speed. The Eulerian density perturbation is in phase with the loop displacement and the volume perturbation. This is because in Eq. (8) the perturbation due to the advection of the equilibrium density dominates over the curvature effect for $\alpha=-4$. However, since $\delta \hat{\rho} / \rho_{0}(R)=-4 A_{m i}$, the Lagrangian density perturbation is in anti-phase with the loop displacement and the volume perturbation.

For a sausage mode $\hat{\rho} / \rho_{0}(R)=2 \mathrm{i}\left|\kappa_{\mathrm{i}}\right| A_{m \mathrm{i}}=$ $2 \mathrm{i} \sqrt{V_{\mathrm{ph}} / V_{\mathrm{Ai}}-1} m A_{m \mathrm{i}}$, which is due to the magnetic pressure perturbation. The density perturbation amplitude lies between 0 and $2 \mathrm{i} \sqrt{(1-\chi) / \chi} m A_{m \mathrm{i}}$. These limits correspond to $V_{\mathrm{ph}}=V_{\mathrm{Ai}}$ (reached in the short wavelength limit) and $V_{\mathrm{ph}}=V_{\mathrm{Ae}}$ (at the cut-offs) respectively.

\section{Comparison with a straight slab model}

We compare our results with that of MHD waves in a straight slab model. Edwin \& Roberts (1982) considered parallel propagating MHD waves in a straight slab with piece-wise constant equilibrium quantities. If we impose the assumptions of the

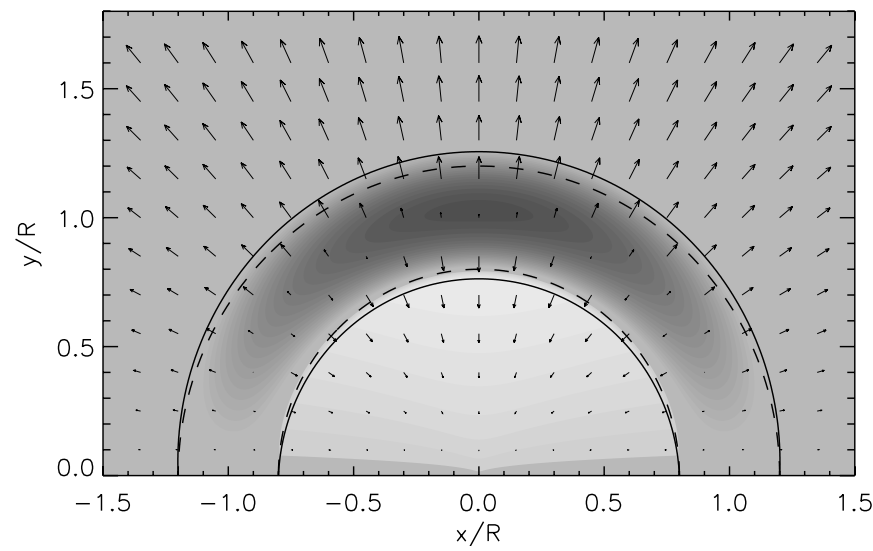

Fig. 9. Density perturbation $\delta \rho / \rho_{0}$ of the fundamental trapped sausage mode with a displacement amplitude of $A_{\mathrm{i}}=-B_{\mathrm{i}}=0.2 \mathrm{i}$. The velocity field is overplotted. The solid (dashed) lines show the (un)perturbed position of the slab edge. The parameters $\chi=0.01$ and $a / R=0.2$.

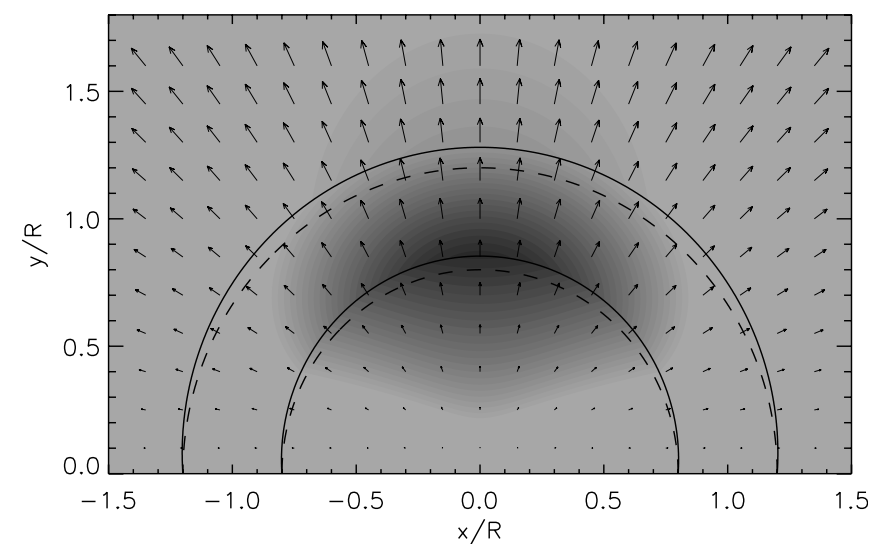

Fig. 10. Density perturbation $\delta \rho / \rho_{0}$ of the fundamental trapped kink mode with a displacement amplitude of $A_{\mathrm{i}}=B_{\mathrm{i}}=0.05$. The velocity field is overplotted. The solid (dashed) lines show the (un)perturbed position of the slab edge. The parameters $\chi=0.2$ and $a / R=0.2$.

internal and external magnetic fields to be identical and the zero plasma- $\beta$ limit, then the dispersion relation for trapped modes in the straight slab model is

$\left\{\begin{array}{l}\tanh \\ \operatorname{coth}\end{array}\right\}\left(\tilde{\kappa}_{\mathrm{i}} \frac{a}{R}\right)=-\frac{\tilde{\kappa}_{\mathrm{i}}}{\left|\tilde{\kappa}_{\mathrm{e}}\right|}$

where $\tilde{\kappa}_{\{i, e\}}$ is of the same form as Eq. (12), but where $m$ is now related to the wave vector component in the direction of the straight equilibrium magnetic field.

We expect the differences between the curved and straight slab models to minimise in two limits: the limit of a slender loop, i.e. $a / R \ll 1$, and in the short wavelength limit, i.e. $m a / R \gg 1$. In the limit of a thin loop the parameter $q$ becomes (see Eq. (17))

$q \approx 1+2 \frac{a}{R} \approx \mathrm{e}^{2 \frac{a}{R}}$

so that dispersion relation (16) tends to dispersion relation (33). This is also the reason why the curves of the two models in Fig. 6 tend to each other for small $a / R$. For instance, the wave solution for leaky modes in the straight slab model in the limit 

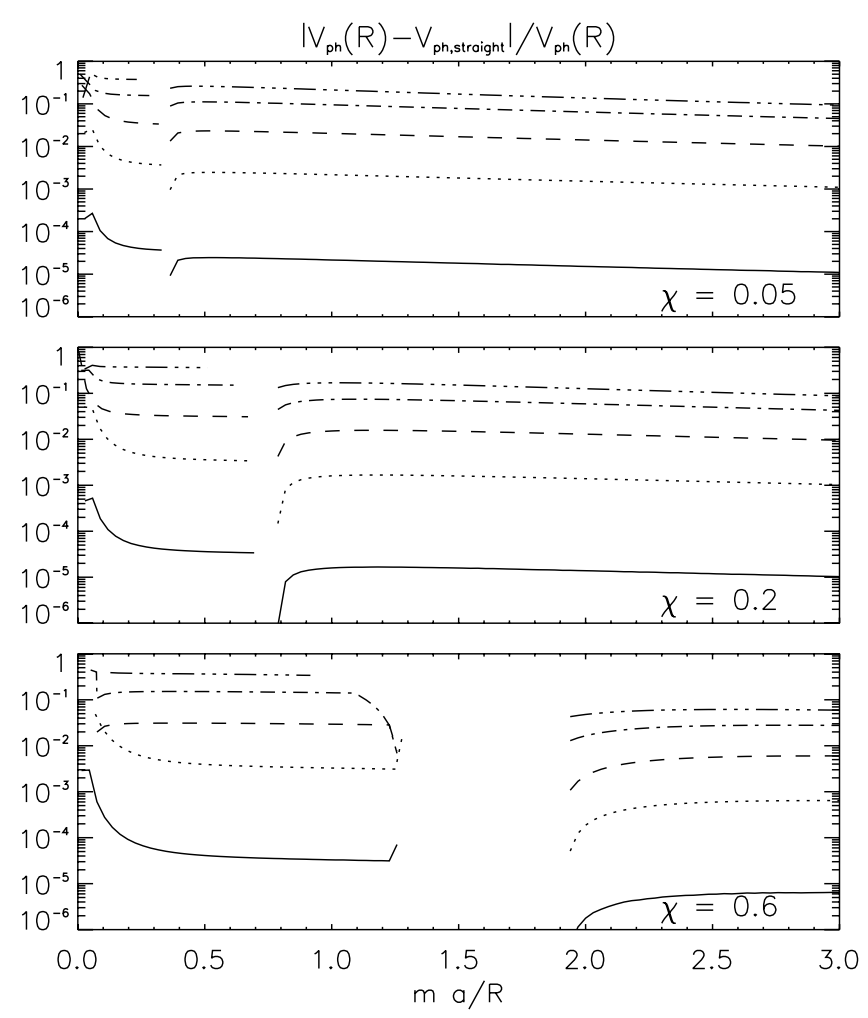

Fig. 11. Relative difference between the phase speeds of the fundamental sausage mode branch $(n=0)$ of the model presented here and the straight slab, plotted as a function of $m a / R$ for three values of $\chi$ (top, $\chi=0.05$, middle, $\chi=0.2$ and bottom, $\chi=0.6$ ) and five values of $a / R$ (solid curve, $a / R=0.01$, dotted curve, $a / R=0.1$, dashed curve, $a / R=0.3$, dot-dashed curve, $a / R=0.6$ and triple dot-dashed curve, $a / R=0.9$ ). The gaps in the curves are where the sausage modes of both models do not share the same behaviour, i.e. both are neither trapped nor leaky.

of small $a / R$ (Cally 1986) is recovered by substituting Eq. (34) into Eq. (26). Furthermore, by taking $r=R+a s$ where $s$ is the distance from the loop axis in units of the loop's minor radius. In the thin loop limit, the solutions close to the loop axis, i.e. $s \sim O(1)$, becomes

$\left(\frac{r}{R}\right)^{ \pm \kappa}=\mathrm{e}^{ \pm \kappa \ln (1+s a / R)} \approx \mathrm{e}^{ \pm \kappa s a / R}$,

and the solution (13) reduces to

$\hat{\xi}_{r} \approx R \sum_{m=1}^{\infty} A_{m} \mathrm{e}^{\kappa s a / R}+B_{m} \mathrm{e}^{-\kappa s a / R}$,

which in turn reduces to the solution for the straight slab model. Similarly, the density perturbation (8) near the loop axis is approximately of the form $\rho / \rho_{0} \approx \partial \xi_{r} / \partial r$, where terms of the order of $a / R$ have been neglected. Using Eq. (36) and considering a kink mode $\left(A_{m}=B_{m}\right)$, we can easily see from Eq. (30) that the density perturbation in the thin loop limit has a zero average across the slab. This is also consistent with the straight slab model. The non-zero average density perturbation of the kink mode is a characteristic of the curved slab model.

In Figs. 11 and 12 we compare the phase speeds of the fundamental kink and sausage mode branches of the curved slab
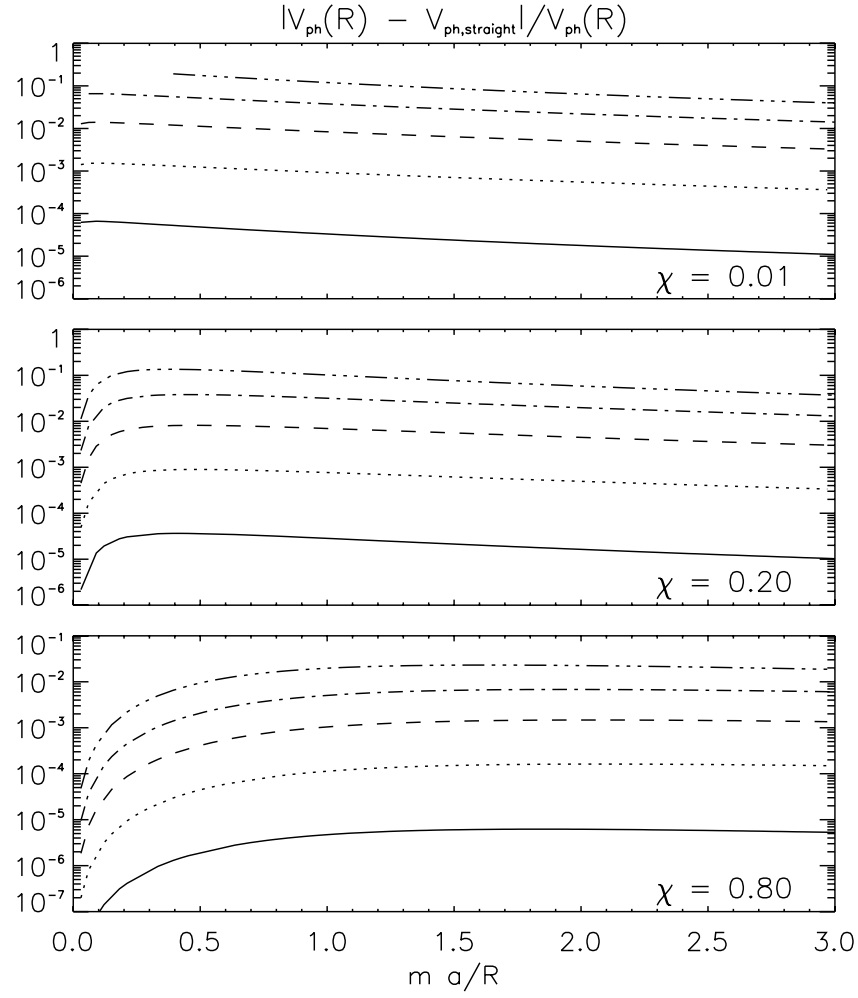

Fig. 12. Relative difference between the phase speeds of the fundamental kink mode branch $(n=-1)$ of the model presented here and the straight slab, plotted as a function of $m a / R$ for three values of $\chi$ (top, $\chi=0.01$, middle, $\chi=0.2$ and bottom, $\chi=0.8$ ) and five values of $a / R$ (solid curve, $a / R=0.01$, dotted curve, $a / R=0.1$, dashed curve, $a / R=0.3$, dot-dashed curve, $a / R=0.6$ and triple dot-dashed curve, $a / R=0.9)$.

model with the straight slab model as a function of $m a / R$ for various values of $\chi$ and $a / R$. As $m a / R$ becomes larger, the difference between the two models decays to zero. Only for values of $a / R$ above roughly 0.5 do the two model differ by more than $5 \%$. This means that for realistic coronal loops, where $a / R$ is of the order of 0.01-0.2, the curved loop model with $\alpha=-4$ and the straight loop model, from the point of view of the mode frequencies, are indistinguishable. Also, from the results it is clear that the sausage and kink modes retain their distinct character as evidence of coupling between the two types of oscillations due to curvature has not been found.

\section{Discussion}

When comparing the calculated quantities with observations, we need to consider the Lagrangian description. We have shown that the Lagrangian density perturbation in a curved loop model has two contributions (see Eq. (10)). The first contribution is due to the magnetic pressure perturbation and appears also in the straight coronal loop model (Edwin \& Roberts 1982). Because the magnetic pressure perturbation is proportional to the radial derivative of $\xi_{r} / r$, its average across the loop is approximately zero for a kink mode (see Fig. 8 as an illustration). Therefore, this term does not contribute to the average density perturbation. The second contribution is due to the loop curvature and is associated with the loop length 
perturbation. Therefore, the density perturbation for a fundamental kink mode, averaged across the loop width, is approximately equal to (using Eq. (10))

$\left\langle\frac{\delta \rho}{\rho_{0}}\right\rangle \approx-2\left\langle\frac{\xi_{r}}{r}\right\rangle \approx-2 \frac{\xi_{r}(R)}{R}$,

where the brackets $\langle f\rangle$ denote a quantity averaged across the width of the loop. The intensity perturbation, $\delta I$, is approximately equal to $2 \rho_{0} \delta \rho$ if the perturbations are small. Therefore, the relation between the intensity and displacement, using Eq. (37) and assuming $\rho_{0}$ does not vary significantly across the slab, is

$$
\frac{\langle\delta I\rangle}{\left\langle I_{0}\right\rangle} \approx 2\left\langle\frac{\delta \rho}{\rho_{0}}\right\rangle \approx-4 \frac{\xi_{r}(R)}{R} .
$$

The observation by Wang \& Solanki (2004) of a vertically polarised fast magnetoacoustic kink wave showed the average intensity and displacement perturbations to be in anti-phase. Wang \& Solanki (2004) reported a displacement amplitude $\xi_{r}(R) / R=0.071$ and an intensity perturbation with an amplitude of approximately unity. This would give an amplitude ratio of -14 , which is much smaller than the predicted value of -4 .

Of course, for such a value of the intensity perturbation amplitude, Eq. (38) is strictly speaking not valid. But at this point we would like to add a note of caution about the value of the intensity perturbation amplitude as reported by Wang \& Solanki (2004). The authors did not take into account the effect of time integration. As the loop oscillates, it spends more time at its extremal positions than in between. The time-integrated intensity is thus larger at those positions compared with the mid-points. The intensity perturbation amplitude due to time integration alone (17 s precisely) would be $\langle\delta I\rangle /\left\langle I_{0}\right\rangle=0.78,0.31$ and 0.19 for loop half-width $a=1000,2000$ and $3000 \mathrm{~km}$ respectively (for a period of $234 \mathrm{~s}$ and displacement amplitude of $7900 \mathrm{~km}$, as deduced by Wang \& Solanki (2004). Therefore, the effect of time-integration can explain for a large part the intensity enhancement at the minimal position. Also, from the data presented by Wang \& Solanki (2004), it is difficult to see a real intensity depletion at the maximal positions (see their Fig. 2). Only two maximal positions of the oscillation are visible. The first coincides with the time of excitation itself and the second is near a data gap due to cosmic ray contamination. Because of these uncertainties, it would therefore be advantageous to have more observational examples of a vertically polarised kink oscillation to confirm the behaviour reported.

But if the vertically polarised kink mode observed by Wang \& Solanki (2004) does possess a significant density perturbation, which is in anti-phase with the displacement, then its amplitude is predicted to be -0.28 . We have shown that for most density profiles, the fast oscillation modes cannot be trapped in the loop and will leak into the external corona by means of wave tunneling. It is of interest to note that Brady \& Arber (2005) have demonstrated this numerically for one such type of density profile, namely $\alpha=0$. Because the wave leakage is expected to be a function of $\alpha$, we expect that the observational measurement of the wave damping to be an additional interesting seismological tool for deducing the equilibrium density profile. Therefore in a future work, we shall investigate, using the governing equations presented here, tunneling fast magnetoacoustic wave modes in curved coronal loop slab models with a density profiles with indices different from $\alpha=-4$.

\section{Conclusions}

We have derived the governing equations for linear vertically polarised fast magnetoacoustic waves in a curved coronal loop. The loop is modeled as a semi-circular magnetic slab in the zero plasma- $\beta$ limit. We summarise our findings as follows:

- The propagatory behaviour of wave modes depends on the transverse equilibrium density profile, which we have modeled as a power law of index $\alpha$. For such a density profile, trapped oscillations are only possible if $\alpha=-4$, which corresponds to a loop with a linear Alfvén speed profile. This case has been studied here in detail. For all other profiles, the mechanism of wave tunneling becomes possible and the wave is expected to leak into the external medium. Therefore, wave leakage is an inherent physical feature of oscillations in coronal loops. A detailed analytical analysis of these other density profiles is reserved for a future paper.

- The Eulerian density perturbation associated with a wave mode depends on three contributions: magnetic pressure, curvature and density advection. The last two terms are new compared with the straight slab model of Edwin \& Roberts (1982). The latter term vanishes when going to the Lagrangian description. The curvature gives rise to a nonzero averaged density perturbation in a fast kink oscillation.

- We have studied the model with a linear Alfvén speed profile $(\alpha=-4)$ in detail and found many similarities with the straight slab model of Edwin \& Roberts (1982). Waves, which exist as sausage and kink mode types, are either trapped body modes or oscillatory (leaky) everywhere. In the long wavelength limit all modes are leaky, except solutions from the fundamental kink mode branch. Evidence for coupling between the fast kink and sausage modes has not been found. This curved slab model reduces to the straight slab model in the limit of a slender loop and for short wavelengths. In fact, for realistic values of the loop width to length ratio, the two models differ by less than 5\%. One important difference, though, is that in the curved slab model, the density perturbation does not average to zero across the loop width, i.e. fast kink oscillations may have significant density perturbations.

The model we have presented, may be improved and extended in several ways. The introduction of a sheared magnetic field is expected to introduce coupling between the Alfvén and magnetoacoustic modes (Goedbloed \& Halberstadt 1994) and it would be interesting to study this in a curved geometry. Also, with a finite plasma- $\beta$, the effect of curvature on slow magnetoacoustic waves may be investigated. Furthermore, a more realistic equilibrium density profile may be introduced, based on gravitational stratification (e.g. Andries et al. 2004; Mendoza-Briceño et al. 2004; Dymova \& Ruderman 2004). This would make the equilibrium two-dimensional. 
Acknowledgements. E.V. is grateful to PPARC for the financial support. V.M.N. acknowledges the support of a Royal Society Leverhulme Trust Senior Research Fellowship. The authors would like to thank T. Arber and G. Rowlands for useful discussions and the referee H. Goedbloed for helpful suggestions.

\section{Appendix A: Derivation of the governing equations}

In the cylindrical system $(r, \phi, z)$, we consider the ideal, gravitationless, zero plasma- $\beta$ MHD equations, which are linearised with respect to small perturbations from the equilibrium, viz.

$$
\begin{aligned}
\rho & =-\boldsymbol{\nabla} \cdot\left(\rho_{0} \boldsymbol{\xi}\right), \\
\rho_{0} \frac{\partial^{2} \boldsymbol{\xi}}{\partial t^{2}} & =(\boldsymbol{\nabla} \times \boldsymbol{B}) \times \frac{\boldsymbol{B}_{\mathbf{0}}}{\mu_{\mathrm{o}}}, \\
\boldsymbol{B} & =\boldsymbol{\nabla} \times\left(\boldsymbol{\xi} \times \boldsymbol{B}_{\mathbf{0}}\right),
\end{aligned}
$$

where $\boldsymbol{\xi}, \boldsymbol{B}$ and $\rho$ are the displacement, magnetic field and density perturbations respectively. The perturbed quantities follow the Eulerian description. Wave propagation can be described in terms of the radial displacement $\xi_{r}$ and the magnetic pressure perturbation $P=B_{0 \phi} B_{\phi} / \mu_{0}$, following a procedure similar to the one described by Sakurai et al. (1991).

The induction Eq. (A.3) and the Lorentz force in the momentum Eq. (A.2) are rewritten as

$\left(\frac{\boldsymbol{B}}{B_{0 \phi}}\right)=\frac{1}{r} \frac{\partial \boldsymbol{\xi}}{\partial \phi}+\left[2 \frac{\xi_{r}}{r}-\boldsymbol{\nabla} \cdot \boldsymbol{\xi}\right] \mathbf{1}_{\phi}$

and

$(\boldsymbol{\nabla} \times \boldsymbol{B}) \times \frac{\boldsymbol{B}_{\mathbf{0}}}{\mu_{\mathrm{o}}}=-\boldsymbol{\nabla} P-\frac{2 P}{r} \mathbf{1}_{r}+\frac{2 P_{0}}{r} \frac{\partial}{\partial \phi}\left(\frac{\boldsymbol{B}}{B_{0 \phi}}\right)$,

respectively. $P_{0}=B_{0 \phi}^{2} / 2 \mu_{\mathrm{o}}$ is the equilibrium magnetic pressure. By eliminating $\boldsymbol{B}$ from Eq. (A.5) using Eq. (A.4), the momentum Eq. (A.2) becomes

$$
\begin{aligned}
\rho_{0} \mathcal{L}_{\mathrm{A}} \boldsymbol{\xi}= & -\boldsymbol{\nabla} P+\frac{P_{0}}{r}\left[2 \boldsymbol{\nabla} \cdot \boldsymbol{\xi}-\frac{P}{P_{0}}-\frac{2}{r} \frac{\partial \xi_{\phi}}{\partial \phi}-4 \frac{\xi_{r}}{r}\right] \mathbf{1}_{r} \\
& +\frac{2 P_{0}}{r} \frac{\partial}{\partial \phi}\left[2 \frac{\xi_{r}}{r}-\boldsymbol{\nabla} \cdot \boldsymbol{\xi}\right] \mathbf{1}_{\phi},
\end{aligned}
$$

with

$\mathcal{L}_{\mathrm{A}}=\frac{\partial^{2}}{\partial t^{2}}-\frac{V_{\mathrm{A}}^{2}}{r^{2}} \frac{\partial^{2}}{\partial \phi^{2}}$,

the Alfvén wave operator. An expression for the dilation $\boldsymbol{\nabla} \cdot \boldsymbol{\xi}$ in terms of $\xi_{r}$ and $P$ is found from the definition of $P$, using Eq. (A.4) and the azimuthal component of Eq. (A.6):

$\boldsymbol{\nabla} \cdot \boldsymbol{\xi}=-\frac{1}{2} \frac{P}{P_{0}}+2 \frac{\xi_{r}}{r}$.

A first equation coupling $\xi_{r}$ and $P$ is derived from the expression $\boldsymbol{\nabla} . \boldsymbol{\xi}-1 / r \partial\left(r \xi_{r}\right) / \partial r$ using Eqs. (A.6)-(A.8). A second equation is derived from the radial component of Eq. (A.6). We find the following set of equations:

$$
\begin{aligned}
\mathcal{L}_{\mathrm{A}} \frac{\partial}{\partial r}\left(\frac{\xi_{r}}{r}\right) & =-\frac{1}{2 r}\left(\mathcal{L}_{\mathrm{A}}-V_{\mathrm{A}}^{2} \frac{\partial^{2}}{\partial z^{2}}\right)\left(\frac{P}{P_{0}}\right), \\
\frac{\partial}{\partial r}\left(\frac{P}{P_{0}}\right) & =-\frac{2 r}{V_{\mathrm{A}}^{2}} \mathcal{L}_{\mathrm{A}} \frac{\xi_{r}}{r} .
\end{aligned}
$$

This set of equations describe Alfvén waves polarised in the $z$-direction and fast magnetoacoustic waves polarised in the $r$-direction. These equations are well-known in fusion plasmas where they describe the z-pinch in the zero plasma- $\beta$ limit (e.g. Goedbloed \& Poedts 2004). Vertically polarised perturbations are of the form $\xi_{r}=\hat{\xi}_{r}(r) \sin (m \phi) \exp (-\mathrm{i} \omega t)$ and $P=\hat{P}(r) \sin (m \phi) \exp (-\mathrm{i} \omega t)$, where $\omega$ is the angular frequency of a mode of azimuthal degree $m$. From Eqs. (A.9) and (A.10), the set of equations describing the fast magnetoacoustic waves then become:

$$
\begin{aligned}
\frac{\mathrm{d}}{\mathrm{d} r}\left(\frac{\hat{\xi}_{r}}{r}\right) & =-\frac{1}{2 r} \frac{\hat{P}}{P_{0}}, \\
\frac{\mathrm{d}}{\mathrm{d} r}\left(\frac{\hat{P}}{P_{0}}\right) & =2 r\left(\frac{\omega^{2}}{V_{\mathrm{A}}^{2}(r)}-\frac{m^{2}}{r^{2}}\right) \frac{\hat{\xi}_{r}}{r} .
\end{aligned}
$$

In the absence of $z$-dependence, the fast magnetoacoustic and Alfvén waves are decoupled. The Alfvén waves are characterised by the dispersion relation $\omega^{2}=m^{2} V_{\mathrm{A}}^{2}(r) / r^{2}$.

\section{References}

Andries, J., Goossens, M., Hollweg, J. V., Arregui, I., \& VanDoorselaere, T. 2004, A\&A, 430, 1109

Aschwanden, M. J., Fletcher, L., Schrijver, C. J., \& Alexander, D. 1999, ApJ, 520, 880

Brady, C. S., \& Arber, T. D. 2005, A\&A, 438, 733

Cally, P. S. 1986, Sol. Phys., 103, 277

Cargill, P. J., Chen, J., \& Garren, D. A. 1994, ApJ, 423, 854

Díaz, A. J., Oliver, R., Ballester, J. L., \& Roberts, B. 2004, A\&A, 424, 1055

Dymova, M. V., \& Ruderman, M. S. 2004, ESA SP, 575, 389

Edwin, P. M., \& Roberts, B. 1982, Sol. Phys., 76, 239

Edwin, P. M., \& Roberts, B. 1983, Sol. Phys., 88, 179

Goedbloed, J. P., \& Halberstadt, G. 1994, A\&A, 286, 275

Goedbloed, H., \& Poedts, S. 2004, Principles of Magnetohydrodynamics (Cambridge University Press)

Heyvaerts, J., \& Priest, E. R. 1983, A\&A, 117, 220

Hollweg, J. V., \& Yang, G. 1988, J. Geophys. Res., 93, 5423

Mendoza-Briceño, C. A., Erdélyi, R., \& Sigalotti, L. Di G. 2004, ApJ, 605,493

Miyagoshi, T., Yokoyama, T., \& Shimojo, M. 2004, PASP, 56, 207

Murawski, K., Selwa, M., \& Nocera, L. 2005, A\&A, 437, 687

Nakariakov, V. M., Ofman, L., DeLuca, E. E., Roberts, B., \& Davila, J. M. 1999, Science, 285, 862

Nakariakov, V. M., Melnikov, V. F., \& Reznikova, V. E. 2003, A\&A, 412, L7

Nakariakov, V. M., \& Verwichte, E. 2005, Living Rev. Solar Phys., 2, 3, URL (cited on 2005-07-05): http://www . livingreviews .org/lrsp-2005-3

Roberts, B. 2000, Sol. Phys., 193, 139

Rosner, R., Low, B. C., \& Holzer, T. E. 1984, ApJ, 271, 820

Sakurai, T., Goossens, M., \& Hollweg, J. V. 1991, Sol. Phys., 133, 247

Selwa, M., Murawski, K., Solanki, S. K., Wang, T. J., \& Tóth, G. 2005, $\mathrm{A} \& \mathrm{~A}$, in press

Smith, J. M., \& Roberts, B. 1997, A\&A, 317, 752

Van Doorselaere, T., DeBosscher, A., Andries, J., \& Poedts, S. 2004, A\&A, 424, 1065

Verwichte, E., Nakariakov, V. M., \& Cooper, F. C. 2005, A\&A, 430, L65

Wang, T. J., \& Solanki, S. K. 2004, A\&A, 421, L33

Williams, D. R., Phillips, K. J. H., Rudawy, P., et al. 2001, MNRAS, 326, 428 